\title{
Morphological identification of right ventricular failure in cases of fatal pulmonary thromboembolism
}

\author{
Tony Fracasso $\cdot$ Heidi Pfeiffer $\cdot$ Cristina Sauerland . \\ Andreas Schmeling
}

Received: 10 June 2010 / Accepted: 28 June 2010 / Published online: 10 July 2010

(C) Springer-Verlag 2010

\begin{abstract}
Pulmonary thromboembolism is a life-threatening event potentially determining right ventricular failure. Even if the pathophysiology of this phenomenon has been widely investigated, no morphological demonstration of right ventricular ischemic damage determining right ventricular failure in cases of fatal pulmonary embolism has been reported till now. We performed an immunohistochemical investigation with the markers fibronectin and C5b-9 in 26 cases of fatal pulmonary thromboembolism $(16$ ㅇ, 10 , mean age 56.4 years), as well as in 25 cases of myocardial infarction $(16+9 \hat{\jmath}$, mean age 60.8 years) and 20 cases of hanging $(3 q, 17 \hat{\jmath}$, mean age 40.8 years). In each case, at least one tissue slide from both cardiac ventricles (free wall of the right ventricle, anterior and/or posterior wall of the left ventricle) was prepared. The reactions were semiquantitatively classified and the groups compared. In the study group, the occurrence of ischemic changes at the right ventricle was significantly higher than in cases of myocardial infarction and global hypoxia due to hanging. The determining aspect of the immunohistochemical examination is the identification of the prevalent ischemic lesion at the right
\end{abstract}

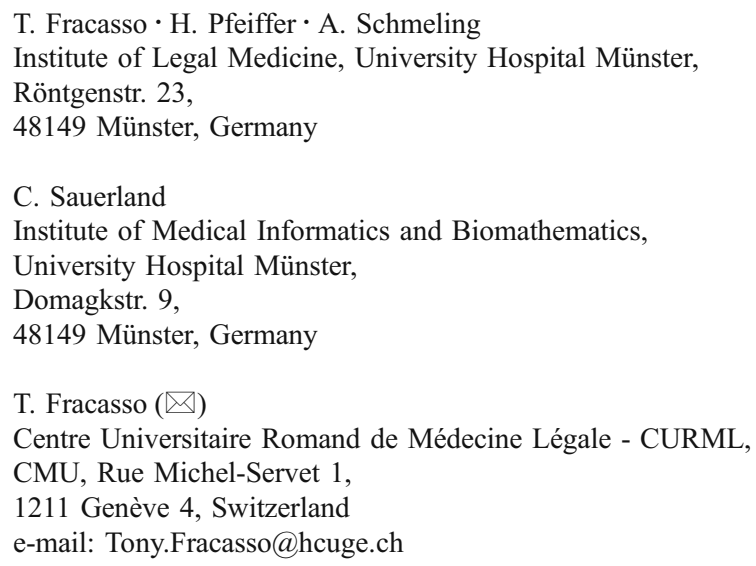

ventricle compared to the left one. This may indicate the primary involvement of the right ventricle thus demonstrating a right ventricular failure.

Keywords Ischemic damage · Pulmonary thromboembolism - Acute pulmonary hypertension - Right ventricular failure - Acute cor pulmonale . Immunohistochemistry

\section{Introduction}

The potentially lethal involvement of the right ventricle in cases of pulmonary thromboembolism (PT) is widely known [1,2] and clinically identifiable with common radiological techniques or biochemical analyses [3-6]. On the contrary, post-mortem identification of right ventricular failure is not possible, and the diagnosis of its occurrence is mostly based on clinical knowledge: pathological signs such as dilatation of the right ventricle at autopsy have been repeatedly described [7,8], but their diagnostic power is doubtful. Not even histological studies aimed at identifying right ventricular damage in cases of pulmonary embolism could demonstrate typical morphological patterns [9-11].

The right ventricular function is commonly compromised when the impedance to right ventricular ejection is acutely increased. Typical is the case of acute pulmonary hypertension following severe PT [12]. PT determines increasing pulmonary pressure in a double way: directly by obstruction of the lumen, indirectly due to reactive vasoconstriction following hypoxemia [13, 14]. As a consequence, the right ventricle reacts with acute dilatation $[15,16]$ and increased coronary perfusion [12] with resulting positive inotropic effect. However, if the pulmonary hypertension persists, these protective mechanisms fail 
Table 1 Study group, cases of fatal pulmonary thromboembolism, $(N=26 ; 16$ +, $10 \AA$, mean age 56.4 years $)$

\begin{tabular}{llllll}
\hline ID Sex Age RV & LV & $\Delta$ & RV & LV & $\Delta$
\end{tabular}

fibronectin fibronectin fibronectin C5b-9 C5b-9 C5b-9

\begin{tabular}{|c|c|c|c|c|c|c|c|}
\hline $1 \mathrm{~F}$ & 71 & 3 & 1 & 2 & 2 & 0 & 2 \\
\hline $2 \mathrm{M}$ & 44 & 3 & 2 & 1 & 0 & 0 & 0 \\
\hline $3 \mathrm{~F}$ & 32 & 2 & 0 & 2 & 0 & 0 & 0 \\
\hline $4 \mathrm{~F}$ & 80 & 3 & 1 & 2 & 1 & 0 & 1 \\
\hline $5 \mathrm{~F}$ & 51 & 2 & 2 & 0 & 0 & 0 & 0 \\
\hline $6 \mathrm{~F}$ & 22 & 2 & 1 & 1 & 0 & 0 & 0 \\
\hline $7 \mathrm{M}$ & 55 & 2 & 1 & 1 & 1 & 0 & 1 \\
\hline $8 \mathrm{M}$ & 65 & 3 & 2 & 1 & 0 & 0 & 0 \\
\hline $9 \mathrm{~F}$ & 49 & 3 & 0 & 3 & 0 & 0 & 0 \\
\hline $10 \mathrm{~F}$ & 51 & 3 & 1 & 2 & 0 & 0 & 0 \\
\hline $11 \mathrm{~F}$ & 49 & 3 & 0 & 3 & 1 & 0 & 1 \\
\hline $12 \mathrm{~F}$ & 72 & 2 & 1 & 1 & 1 & 0 & 1 \\
\hline $13 \mathrm{M}$ & 83 & 2 & 1 & 1 & 0 & 0 & 0 \\
\hline $14 \mathrm{~F}$ & 80 & 3 & 1 & 2 & 0 & 0 & 0 \\
\hline $15 \mathrm{M}$ & 72 & 3 & 0 & 3 & 0 & 0 & 0 \\
\hline $16 \mathrm{M}$ & 43 & 3 & 1 & 2 & 0 & 0 & 0 \\
\hline $17 \mathrm{~F}$ & 84 & 3 & 2 & 1 & 0 & 0 & 0 \\
\hline $18 \mathrm{~F}$ & 75 & 3 & 1 & 2 & 0 & 0 & 0 \\
\hline $19 \mathrm{~F}$ & 48 & 2 & 0 & 2 & 2 & 0 & 0 \\
\hline $20 \mathrm{M}$ & 23 & 3 & 3 & 0 & 1 & 0 & 1 \\
\hline $21 \mathrm{~F}$ & 22 & 2 & 2 & 0 & 2 & 0 & 2 \\
\hline $22 \mathrm{M}$ & 38 & 3 & 1 & 2 & 0 & 0 & 0 \\
\hline $23 \mathrm{~F}$ & 87 & 2 & 1 & 1 & 0 & 0 & 0 \\
\hline $24 \mathrm{~F}$ & 82 & 2 & 0 & 2 & 0 & 0 & 0 \\
\hline $25 \mathrm{M}$ & 31 & 2 & 0 & 2 & 0 & 0 & 0 \\
\hline $26 \mathrm{M}$ & 57 & 2 & 1 & 1 & 0 & 0 & 0 \\
\hline
\end{tabular}

and contribute to ischemic damage: the right ventricular dilatation, in fact, affects the left ventricular diastolic compliance determining a decrease in the left ventricular output with subsequently reduced coronary perfusion of the right ventricle and ischemic damage due to the combination of lower coronary supply and increased demand for oxygen $[3,17-19]$. Even if this theory is generally accepted, morphological evidence of ischemic damage is still missing: Iwadate et al. [7, 11] could not demonstrate ischemic changes at the right ventricle in cases of PT, but could observe a tendency to increased macrophage infiltrates in cases of PT. The variable occurrence of myocarditis/endocarditis at the right and left ventricle in cases of lethal PT was also observed by Begieneman et al. [20].

We have recently proposed a method [21] that, in cases of severe pulmonary fat embolism, is able to detect prevalent ischemic necrosis at the right ventricle compared to the left. This method is based on the evaluation of the expression of the immunohistochemical markers fibronectin and the terminal complement complex C5b-9 in the myocardium of both ventricles. Fibronectin is a wellstudied glycoprotein present in the extracellular matrix where it plays a major role in the processes of cell migration, adhesion and wound healing [22]. In legal medicine, it has been extensively investigated in the field of the wound age estimation, with a special application for the detection of the vitality of skin wounds [23]. Recently, Bohnert et al. [24] have proposed a further application for the evaluation of vitality in burnt bodies. In cases of cardiac ischemic damage, fibronectin diffuses into the necrotic cardiac myocytes where it probably binds to actin filaments, thus granting tensile strength of the infarcted ventricle before deposition of new collagen occurs [25].

The terminal complement complex C5b-9 accumulates on the surface of damaged cardiac cells and promotes the formation of pores on the cellular membrane [26]. C5b-9 can be detected if the survival time after onset of the cardiac ischemia is longer than $30-40 \mathrm{~min}$ [27]; the

Table 2 Cases of heart infarction $(N=25,16$,, $9 \hat{\jmath}$, mean age 60.8 y)

\begin{tabular}{|c|c|c|c|c|c|c|c|}
\hline ID Sex & Age & $\begin{array}{l}\text { RV } \\
\text { Fibronectin }\end{array}$ & $\begin{array}{l}\text { LV } \\
\text { Fibronectin }\end{array}$ & $\begin{array}{l}\Delta \\
\text { Fibronectin }\end{array}$ & $\begin{array}{l}\text { RV } \\
\text { C5b- } \\
9\end{array}$ & $\begin{array}{l}\text { LV } \\
\text { C5b- } \\
9\end{array}$ & $\begin{array}{l}\Delta \\
\text { C5b- } \\
9\end{array}$ \\
\hline $1 \mathrm{~F}$ & 61 & 2 & 2 & 0 & 0 & 2 & -2 \\
\hline $2 \mathrm{~F}$ & 40 & 2 & 2 & 0 & 0 & 0 & 0 \\
\hline $3 \mathrm{~F}$ & 41 & 2 & 3 & -1 & 0 & 0 & 0 \\
\hline $4 \mathrm{~F}$ & 40 & 2 & 2 & 0 & 0 & 0 & 0 \\
\hline $5 \mathrm{~F}$ & 46 & 1 & 1 & 0 & 0 & 0 & 0 \\
\hline $6 \mathrm{~F}$ & 81 & 2 & 3 & -1 & 2 & 2 & 0 \\
\hline $7 \mathrm{M}$ & 43 & 2 & 2 & 0 & 0 & 0 & 0 \\
\hline $8 \mathrm{~F}$ & 82 & 2 & 2 & 0 & 0 & 0 & 0 \\
\hline $9 \mathrm{~F}$ & 76 & 1 & 2 & -1 & 0 & 0 & 0 \\
\hline $10 \mathrm{M}$ & 67 & 2 & 3 & -1 & 0 & 2 & -2 \\
\hline $11 \mathrm{M}$ & 70 & 2 & 3 & -1 & 1 & 1 & 0 \\
\hline $12 \mathrm{M}$ & 66 & 2 & 2 & 0 & 0 & 0 & 0 \\
\hline $13 \mathrm{~F}$ & 53 & 2 & 2 & 0 & 0 & 0 & 0 \\
\hline $14 \mathrm{M}$ & 38 & 3 & 3 & 0 & 0 & 0 & 0 \\
\hline $15 \mathrm{~F}$ & 58 & 2 & 2 & 0 & 0 & 0 & 0 \\
\hline $16 \mathrm{~F}$ & 87 & 2 & 0 & 2 & 0 & 2 & -2 \\
\hline $17 \mathrm{~F}$ & 80 & 2 & 3 & -1 & 1 & 1 & 0 \\
\hline $18 \mathrm{~F}$ & 46 & 1 & 1 & 0 & 0 & 0 & 0 \\
\hline $19 \mathrm{M}$ & 41 & 3 & 3 & 0 & 0 & 2 & -2 \\
\hline $20 \mathrm{M}$ & 60 & 2 & 2 & 0 & 0 & 0 & 0 \\
\hline $21 \mathrm{~F}$ & 41 & 2 & 3 & -1 & 0 & 0 & 0 \\
\hline $22 \mathrm{M}$ & 47 & 1 & 3 & -2 & 0 & 2 & -2 \\
\hline $23 \mathrm{~F}$ & 88 & 2 & 3 & -1 & 0 & 1 & -1 \\
\hline $24 \mathrm{~F}$ & 82 & 2 & 2 & 0 & 0 & 0 & 0 \\
\hline $25 \mathrm{M}$ & 87 & 1 & 3 & -2 & 1 & 2 & -1 \\
\hline
\end{tabular}


intrasarcolemmal accumulation of fibronectin is detectable even earlier [28].

The hypothesis at the base of the present study is that primary right heart ischemia due to pulmonary hypertension, in cases of PT, can be distinguished from primary left ventricular failure due to myocardial infarction and from heart damage due to global hypoxia. The nature of the myocardial lesions, in cases of acute myocardial infarction, does not differ from the cardiac damage following pulmonary embolism, as both are ischemic; nevertheless, our hypothesis is that from a morphological point of view they can be distinguished: in cases of myocardial infarction, the left ventricle is more affected than the right one because of its major oxygen demand and the relatively minor functional reserves, if compared to the right one. For these reasons, the extension and the localization of the ischemic cardiac damage should allow a good differentiation between primary lesions following pulmonary hypertension due to pulmonary embolism (prevalently localised at the right ventricle) and acute ischemic damage following acute myocardial infarction (prevalently localised at the left ventricle or equally distributed in the myocardium of both ventricles).

Finally, the comparison with a third group (cases of hanging) is useful to differentiate the pattern of ischemic lesions from agonal necroses due to terminal global hypoxia. In this group, fresh cardiac damage should be less extensive than in cases of ischemia and show a similar distribution in the two ventricles or a predilection for the left one.

\section{Materials and methods}

Our material constituted of 26 cases of fatal pulmonary thromboembolism (study group, $16 \uparrow, 10 \hat{\jmath}$, mean age 56.4 years), 25 cases of acute myocardial infarction (group 2,16 ㅇ, $9 \hat{\jmath}$, mean age 60.8 years) and 20 cases of hanging (group 3, $3 q, 17 \hat{\jmath}$, mean age 40.8 years). All these cases were collected at the Institute of Legal Medicine of the University Hospital in Münster and retrospectively investigated. In each case, information about circumstances of death, as well as autopsy record, was available. An overview of the investigated cases is given in Tables 1, 2 and 3. In each case, immunohistochemical reactions with the antibodies fibronectin (Polyclonal Rabbit Anti-Human, DAKO Deutschland GmbH, Hamburg, Germany) and C5b9 (Monoclonal Mouse Anti-Human, DAKO Deutschland $\mathrm{GmbH}$, Hamburg, Germany) of at least one tissue slide from both cardiac ventricles (free wall of the right ventricle $(\mathrm{RV})$, anterior and/or posterior wall of the left ventricle (LV)) were prepared as described elsewhere [19, 20]. A blind investigation of the slides was performed by two

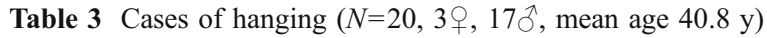

\begin{tabular}{|c|c|c|c|c|c|c|c|}
\hline ID Sex & Age & $\begin{array}{l}\mathrm{RV} \\
\text { fibronectin }\end{array}$ & $\begin{array}{l}\text { LV } \\
\text { fibronectin }\end{array}$ & $\begin{array}{l}\Delta \\
\text { fibronectin }\end{array}$ & $\begin{array}{l}\text { RV } \\
\text { C5b-9 }\end{array}$ & $\begin{array}{l}\text { LV } \\
\text { C5b-9 }\end{array}$ & $\begin{array}{l}\Delta \\
\text { C5b-9 }\end{array}$ \\
\hline $1 \mathrm{M}$ & 42 & 2 & 2 & 0 & 0 & 0 & 0 \\
\hline $2 \mathrm{M}$ & 44 & 0 & 0 & 0 & 0 & 0 & 0 \\
\hline $3 \mathrm{M}$ & 56 & 1 & 2 & -1 & 0 & 0 & 0 \\
\hline $4 \mathrm{~F}$ & 46 & 0 & 0 & 0 & 0 & 0 & 0 \\
\hline $5 \mathrm{M}$ & 23 & 0 & 1 & -1 & 0 & 0 & 0 \\
\hline $6 \mathrm{M}$ & 44 & 0 & 0 & 0 & 0 & 0 & 0 \\
\hline $7 \mathrm{M}$ & 54 & 2 & 2 & 0 & 0 & 0 & 0 \\
\hline $8 \mathrm{~F}$ & 55 & 2 & 2 & 0 & 0 & 0 & 0 \\
\hline $9 \mathrm{~F}$ & 43 & 2 & 2 & 0 & 0 & 0 & 0 \\
\hline $10 \mathrm{M}$ & 42 & 2 & 2 & 0 & 0 & 0 & 0 \\
\hline $11 \mathrm{M}$ & 35 & 1 & 1 & 0 & 0 & 0 & 0 \\
\hline $12 \mathrm{M}$ & 56 & 2 & 2 & 0 & 0 & 0 & 0 \\
\hline $13 \mathrm{M}$ & 49 & 2 & 2 & 0 & 0 & 0 & 0 \\
\hline $14 \mathrm{M}$ & 20 & 0 & 1 & -1 & 0 & 0 & 0 \\
\hline $15 \mathrm{M}$ & 35 & 2 & 2 & 0 & 0 & 0 & 0 \\
\hline $16 \mathrm{M}$ & 21 & 0 & 0 & 0 & 0 & 0 & 0 \\
\hline $17 \mathrm{M}$ & 40 & 2 & 2 & 0 & 0 & 0 & 0 \\
\hline $18 \mathrm{M}$ & 41 & 2 & 2 & 0 & 0 & 0 & 0 \\
\hline $19 \mathrm{M}$ & 38 & 2 & 2 & 0 & 0 & 0 & 0 \\
\hline $20 \mathrm{M}$ & 31 & 2 & 2 & 0 & 0 & 0 & 0 \\
\hline
\end{tabular}

different observers with final consensual evaluation. The positive reactions were semiquantitatively classified as follows [21]:

Grade 0: negative

Grade 1: single cells necrosis

Grade 2: group cells necrosis

Grade 3: diffuse necrosis

The grade of necrosis in each ventricle for each antibody in the study group was compared to the corresponding value of the groups 2 and 3 (grade of necrosis RV fibronectin study group versus grade of necrosis RV fibronectin groups 2 and 3; grade of necrosis RV C5b-9 study group versus grade of necrosis RV C5b-9 groups 2 and 3; grade of necrosis LV fibronectin study group versus grade of necrosis LV fibronectin groups 2 and 3; grade of necrosis LV C5b-9 study group versus grade of necrosis RV C5b-9 groups 2 and 3).

The difference of the grade of the necrosis between right and left ventricle was calculated in each case $(\Delta=$ grade necrosis RV - grade necrosis LV) with both antibodies. A comparison of these differences between study group and groups 2 and 3 was performed. Groups 2 and 3 were finally compared to each other.

Statistical analyses were performed using SAS 9.2 (SAS Institute Inc., Cary, NC). 
Fisher's exact test was used for all comparisons between the investigated groups. The result was considered significant in case of the two-sided $p<0.05$.

\section{Results}

The comparison of the grade of necrosis in the right ventricle showed a higher expression of the antibody fibronectin in the study group compared to both cases of acute myocardial infarctions and hanging $(p<0.0001)$. Fibronectin was more expressed in the right ventricle in cases of heart infarction compared to hanging $(p<0.01)$.

The terminal complement portion C5b-9 was more expressed at the right ventricle in cases of PT compared to cases of hanging, in which it was never detected $(p<0.035)$. A similar expression was detected in comparison to myocardial infarctions.

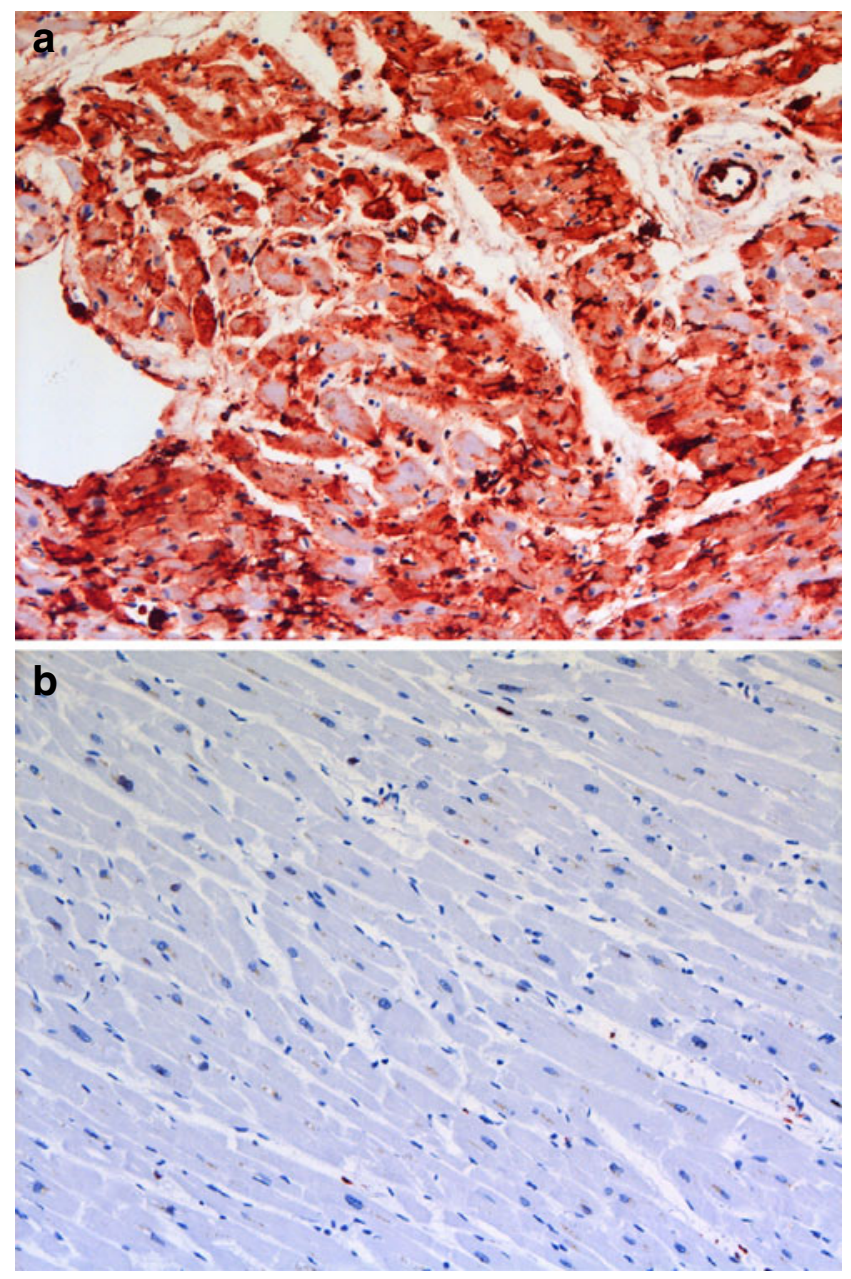

Fig. 1 Immunohistochemical reaction with the antiboby fibronectin, $\times 200$. a Massive positive reaction indicating diffuse ischemic damage at the right ventricle, grade 3 . b Same case, negative reaction at the myocardium of the left ventricle, grade 0 . In this case $\Delta=3$

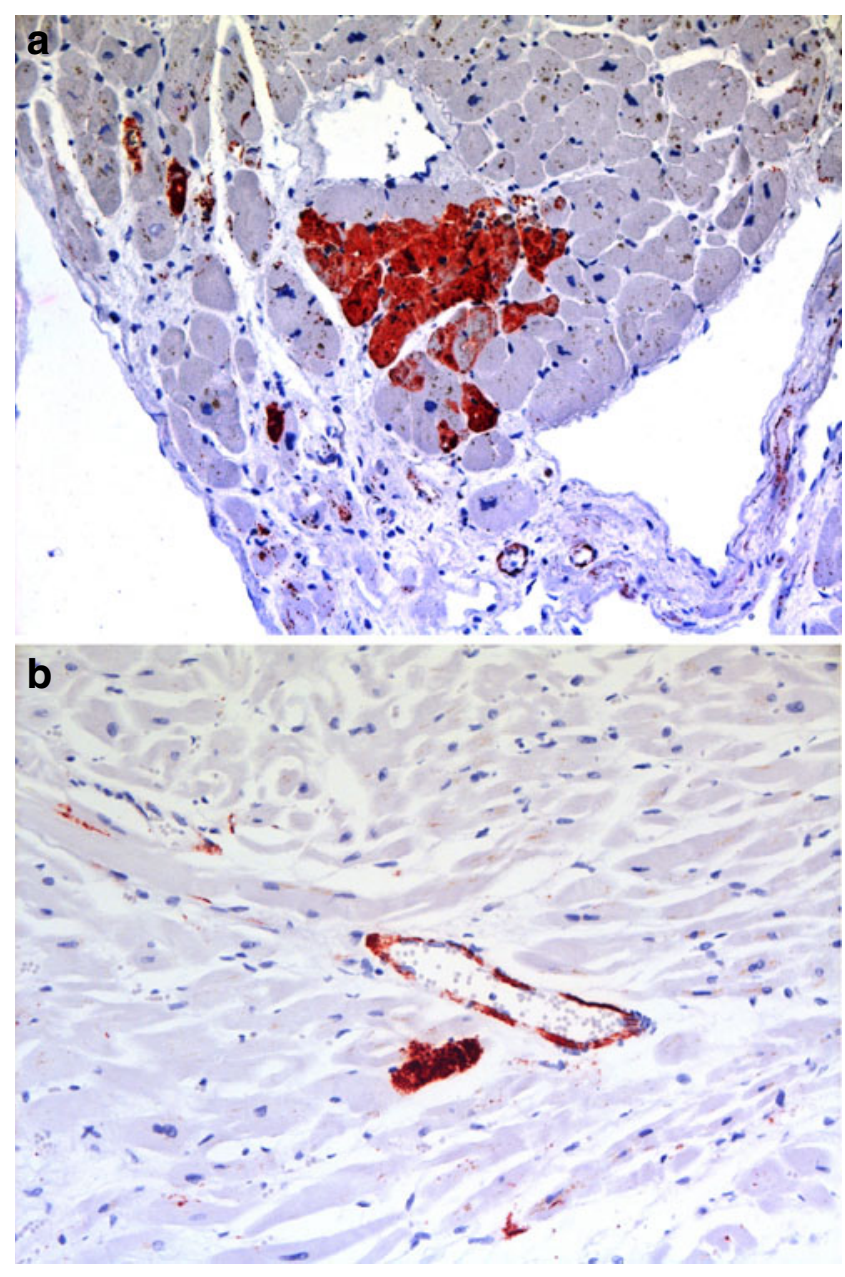

Fig. 2 Immunohistochemical reaction with the antiboby C5b-9, $\times 200$. a Positive reaction indicating group cells necrosis at the right ventricle, grade 2. b Same case, single cells necrosis at the myocardium of the left ventricle, grade 1 . In this case $\Delta=1$

The comparison of the grade of necrosis in the left ventricles showed a prevalent involvement of this ventricle in both groups 2 and 3 compared to the study group, with fibronectin $(p<0.0001$ and $p<0.0059$, respectively). The comparison between groups 2 and 3 showed a higher expression at the left ventricle in cases of heart infarction $(p<0.0013)$.

The terminal complement portion C5b-9 was expressed at the left ventricle only in cases of myocardial infarction. The differences with the study group and the group hanging are statistically significant ( $p<0.001$ and $p<0.004$, respectively).

The comparison of the difference of the grade of the necrosis between right and left ventricle with the antibody fibronectin ( $\Delta$ fibronectin) showed a statistically significant difference between the study group and both groups 2 and 3 $(p<0.0001)$. Similar was instead the distribution of the $\Delta$ fibronectin between groups 2 and 3 .

The comparison of the difference of the grade of the necrosis between right and left ventricle with the antibody 
C5b-9 ( $\Delta$ C5b-9) showed a statistically significant difference between the study group and both groups 2 and $3(p<0.0004$ and $p<0.347$, respectively). This parameter showed a different distribution also between cases of heart infarction and hanging $(p<0.0123)$.

\section{Discussion}

In this study, we demonstrated the presence of prevalent ischemic cardiac damage at the right ventricle in cases of fatal pulmonary thromboembolism compared to cases of acute myocardial infarction and cases of global hypoxia due to hanging.

Interestingly, fresh necrosis at the right ventricle was detected in $100 \%$ of the cases of PT and myocardial infarction, as well as in $70 \%$ of the cases of hanging with the antibody fibronectin. The antibody C5b-9 was expressed in $27 \%$ of the PT cases, in $16 \%$ of the group myocardial infarction and was never expressed in cases of hanging. Such a difference in the expression of these markers is not surprising: Fibronectin is considered a trustful marker for detection of very early cardiac ischemic necrosis [28], while C5b-9 is known to react later [27, 28]. The weaker reaction of the terminal complement complex probably depends on the short duration of agony since the occurrence of the ischemic damage detectable with fibronectin. Clinical experience supports this hypothesis: one of the most important prognostic parameter in patients with PT is the elevation of the troponins as early marker of cardiac ischemic damage: even patients with severe PT have a good prognosis if troponins are normal at the time of hospitalisation [29], this phase corresponds to the time in which the compensative dilatation of the right ventricle is still effective and ischemia has not yet occurred; on the other hand, even a moderate rise of the blood troponin level correlates with short-term poor outcome [30], indicating a very rapid death, after beginning of the ischemic damage. This may also be the explanation for the missed detection of ischemic damage in previous studies where unspecific signs or late markers of ischemia had been applied [11, 20]. At the light of our results, the described macrophage activation and the occurrence of myocarditis/endocarditis $[11,20]$ may be interpreted as an inflammatory reaction occurring after non-lethal ischemic damage, possibly in cases of recurrent transitory PT. These inflammatory changes may reflect the response to PT-induced ischemic changes before occurrence of the lethal massive embolism.

For the morphological identification of cardiac ischemia determining right heart failure, the discriminating element is the evaluation of the difference between RV and LV. The simple detection of necrosis in the right ventricle, in fact, cannot be considered a reliable sign of primary involvement of the RV as the most common cause of right ventricular failure is the failure of the left ventricle [31]: in cases of myocardial infarction with primary involvement of the left ventricle, fresh cardiac necrosis regularly occurs also at the right chamber. The comparison between right and left ventricle is a simple method to verify if necrosis is more detectable at the right chamber (as in the investigated cases of PT), at the left ventricle (as in cases of myocardial infarction) or is homogeneously distributed (as in cases of hanging). Therefore, we propose that only if the necrosis is prevalently localised at the $\mathrm{RV}$, one can consider the primary involvement of the RV in the determination of death. In other words, if the $\Delta$ is bigger than 0 , a primary right ventricular failure can be taken into account (Figs. 1 and 2).

If compared to cases of pulmonary fat embolism [21], the results of the present study more clearly show the correlation with the pulmonary embolism and the occurrence of ischemic damage at the right ventricle leading to failure and death.

It can be concluded that the presented method is able to detect a prevalent ischemic damage at the right ventricle in cases of fatal PT. In such cases, the cause of death is attributable to acute right ventricular failure. This study confirms the efficacy of this method as a tool for the postmortem identification of right ventricular failure due to ischemia in cases of fatal pulmonary embolism.

\section{References}

1. Goldhaber SZ, Visani L, De Rosa M (1999) Acute pulmonary embolism: clinical outcomes in the International Cooperative Pulmonary Embolism Registry (ICOPER). Lancet 353:1386-1389

2. Torbicki A, van Beek EJR, Charbonnier B et al (2000) Guidelines on diagnosis and management of acute pulmonary embolism. Eur Heart J 21:1301-1336

3. Vlahakes GJ, Turley K, Hoffman JIE (1981) The pathophysiology of failure in acute right ventricular hypertension: hemodynamic and biochemical correlations. Circulation 63:87-95

4. Ribeiro A, Lindmarker P, Juhlin-Dannfelt A et al (1997) Echocardiography Doppler in pulmonary embolism: right ventricular dysfunction as a predictor of mortality rate. Am Heart J 134:479-487

5. Kasper W, Konstantinides S, Geibel A et al (1997) Prognostic significance of right ventricular afterload stress detected by echocardiography in patients with clinically suspected pulmonary embolism. Heart 77:346-349

6. Kjaergaard J, Krogsgaard Schaadt B, Lund JO, Hassager C (2006) Quantitative measures of right ventricle dysfunction by echocardiography in the diagnosis of acute nonmassive pulmonary embolism. J Am Soc Echocardiogr 19:1264-1271

7. Iwadate K, Tanno K, Doi M et al (2001) Two cases of right ventricular ischemic injury due to massive pulmonary embolism. For Sci Int 116:189-195

8. Ro A, Kageyama N, Tanifuji T, Fukunaga T (2008) Pulmonary thromboembolism: overview and update from medicolegal aspects. Leg Med 10:57-71 
9. Putnoky J, Farkas K (1933) Vergleichende pathologischhistologische Untersuchung des Herzmuskels bei 1009 Obduktionen, unter besonderer Beachtung der Fälle von Thrombosen und Embolien. Virchows Arch 287:400-404

10. Brandt G, Sasse P, Gunselmann W (1976) Mineralgehalt und Morphologie der Herzmuskulatur bei unterschiedlicher Todesursache. Virchows Arch A Path Anat Histol 369:335-345

11. Iwadate K, Doi M, Tanno K et al (2003) Right ventricular damage due to pulmonary embolism: examination of the number of infiltrating macrophages. For Sci Int 134:147-153

12. Hurford WE, Zapol WM (1988) The right ventricle and critical illness: a review of anatomy, physiology, and clinical evaluation of its functions. Intensive Care Med 114:448-457

13. Von Euler US, Liljestrand G (1946) Observations on the pulmonary arterial blood pressure in the cat. Acta Physiol Scand $12: 301-320$

14. Ward JPT, Aaronson PI (1999) Mechanism of hypoxic pulmonary vasoconstriction: can anyone be right? Resp Physiol 115:261-271

15. Anrep G (1912) On the part played by the suprarenals in the normal vascular reactions of the body. J Physiol 45:307-317

16. Hurford WE, Barlai-Kovach M et al (1987) Canine biventricular performance during acute progressive pulmonary microembolisation: regional myocardial perfusion and fatty acid uptake. J Crit Care 2:270-281

17. Dack S, Master AM, Horn $H$ et al (1949) Acute coronary insufficiency due to pulmonary embolism. Am J Med 7:464-477

18. Gold FL, Bache RJ (1982) Transmural right ventricular blood flow during acute pulmonary artery hypertension in sedated dog. Evidence for subendocardial ischemia despite residual vasodilator reserve. Circ Res 51:196-204

19. Watts JA, Zagorski J, Gellar MA et al (2006) Cardiac inflammation contributes to right ventricular dysfunction following experimental pulmonary embolism in rats. J Mol Cell Cardiol 41:296307

20. Bogieneman MPV, van de Goot FRW, van der Bilt IAC et al (2008) Pulmonary embolism causes endomyocarditis in the human heart. Heart 94:450-456
21. Fracasso T, Karger B, Pfeiffer H, Sauerland C, Schmeling A (2009) Immunohistochemical identification of prevalent right ventricular ischemia causing right heart failure in cases of pulmonary fat embolism. Int J Legal Med. doi:10.1007/s00414009-0382-3

22. Mamuya WS, Brecher P (1992) Fibronectin expression in normal and hypertrophic rat heart. J Clin Invest 89:392-401

23. Betz P, Nerlich A, Wilske J, Tübel J, Penning R, Eisenmenger W (1993) The immunohistochemical localisation of alpha1antichymotrypsin and fibronectin and its meaning for the determination of the vitality of human skin wounds. Int J Legal Med 105:223-227

24. Bohnert M, Anderson J, Rotschild MA, Böhm J (2010) Immunohistochemical expression of fibronectin in the lungs of fire victims proves intravital reaction in fatal burns. Int $\mathrm{J}$ Legal Med. doi:10.1007/s00414-010-0437-5

25. Casscells W, Kimura H, Sanchez JA, Yu Z-X, Ferrans VJ (1990) Immunohistochemical study of fibronectin in experimental myocardial infarction. Am J Pathol 137:801-810

26. Edston E, Kawa K (1995) Immunohistochemical detection of early myocardial infarction. An evaluation of antibodies against the terminal complement complex (C5b-9). Int J Legal Med 108:27-30

27. Thomsen H, Held H (1995) Immunohistochemical detection of C5b-9 $9_{(\mathrm{m})}$ in myocardium: an aid in distinguishing infarctioninduced ischemic heart muscle necrosis from other forms of lethal myocardial injury. Forensic Sci Int 71:87-95

28. Ortmann C, Pfeiffer H, Brinkmann B (2000) A comparative study on the immunohistochemical detection of early myocardial damage. Int J Legal Med 113:215-220

29. Aujesky D, Hughes R, Jiménez D (2009) Short-term prognosis of pulmonary embolism. J Thromb Haemost 7:318-321

30. Becattini C, Vedovati MC, Agnelli G (2007) Prognostic value of troponins in acute pulmonary embolism: a meta-analysis. Circulation 116:427-433

31. Barnard D, Alpert JS (1987) Right ventricular function in health and disease. Curr Probl Cardiol 12:423-449 